\title{
Hypersensitivity pneumonitis and its correlation with ambient air pollution in urban India
}

\author{
To the Editor:
}

Hypersensitivity pneumonitis (HP) is an immune-mediated lung disease (ILD) caused by sensitisation to inhaled organic antigens or low molecular weight chemical compounds. Recently, there has been increased awareness of environmental factors associated with HP [1]. It has been postulated that when genetically susceptible individuals ("first hit") are exposed to an inciting antigen ("second hit") they develop HP [2]. The first hit is essential, as not all who are exposed to an inciting antigen develop disease. The second hit or the inciting antigen may be bacterial, protozoal, animal or insect protein, or rarely a chemical compound. Certain viral infections and high pesticide exposures may be risk factors for development of HP, perhaps by making the lung more susceptible to organic antigen exposure (facilitating a second hit) $[3,4]$.

The role of ambient air pollution as a promoting factor for the development of HP has not been studied. Levels of air pollutants have increased in urban areas over the past decade [5]. Particulate matter $<2.5 \mu \mathrm{m}$ in diameter (PM2.5) can reach the alveoli. Among measures of air pollution, PM2.5 levels have the greatest health impact; higher PM2.5 levels are associated with early mortality [6]. Recently, the role of particulate matter $<10 \mu \mathrm{m}$ in diameter (PM10) has been associated with increased rate of decline of lung functions in patients with idiopathic pulmonary fibrosis (IPF) [7]. However, the role of air pollution in HP has not been studied.

Recent data from the ILD-India registry showed a high proportion of patients with new-onset ILD in India were diagnosed with HP [1]. Since the majority of patients in the ILD-India registry were recruited from urban areas, we hypothesised that air pollution in cities is a contributing factor to development of HP. The aim of this study was to determine whether there is an association between the proportions of HP cases in various Indian cities and ambient air pollution levels measured by PM2.5 using prospective data from patients diagnosed with HP in the ILD-India registry [1].

The ILD-India registry recruited consecutive consenting patients with ILD from 27 centres across 19 cities in India from March 2012 to June 2015. The study was approved by the institutional review board and clinical trial registry of India (CTRI/2013/05/003674). Nearly half of 1084 patients with new-onset ILD living in India had a clinical diagnosis of HP [1]. We analysed data gathered from patients seen at centres that recruited $\geqslant 10$ patients diagnosed with ILD from cities where PM2.5 levels during the period of study were available (figure 1a and b). Patients residing in urban areas from 19 centres across 11 cities were included. In the ILD-India registry, $842(78 \%)$ out of 1084 patients diagnosed with ILD were residing in cities with PM2.5 levels during the study period available for analysis. Among those patients, 386 (45.8\%) out of 842 were diagnosed with HP. The annual mean PM2.5 levels were obtained retrospectively from data provided by the state pollution control boards to the World Health Organization [8]. PM2.5 levels in various cities were either measured directly or derived from PM10 levels. Stata 14.0 (StataCorp LP, College Station, TX, USA) was used for analyses. Spearman's rank correlation test was used to obtain the coefficient of correlation. A scatter diagram with the best fitting line for linear regression (figure 1c) was generated and Lowess smoothing (figure 1d) was created. Multiple logistic regression analysis was performed to determine the association between the percentage of HP cases and PM2.5 level (continuous variable) after adjusting for age, sex, exposure to birds, moulds, desert coolers, air conditioners and smoking.

@ERSpublications

A higher proportion of patients diagnosed with hypersensitivity pneumonitis lives in cities with higher levels of air pollutants. Further studies are warranted to investigate the significance of the association of air pollution to possible causality of HP. http://ow.ly/zH3U30mtocl

Cite this article as: Singh S, Collins BF, Bairwa M, et al. Hypersensitivity pneumonitis and its correlation with ambient air pollution in urban India. Eur Respir J 2019; 53: 1801563 [https://doi.org/10.1183/ 13993003.01563-2018]. 
a)

\begin{tabular}{lccccc}
\hline & City & $\begin{array}{c}\text { HP } \\
\text { cases }\end{array}$ & $\begin{array}{c}\text { Total } \\
\text { ILD cases }\end{array}$ & $\begin{array}{c}\text { ILD cases } \\
\text { with HP }\end{array}$ & $\begin{array}{c}\text { PM2.5 } \\
\boldsymbol{\mu g} \cdot \mathbf{m}^{-3}\end{array}$ \\
\hline $\mathbf{1}$ & Varanasi & 38 & 63 & $60.3 \%$ & 74 \\
$\mathbf{2}$ & Delhi & 68 & 117 & $58.1 \%$ & 122 \\
$\mathbf{3}$ & Lucknow & 9 & 17 & $52.9 \%$ & 113 \\
$\mathbf{4}$ & Kanpur & 66 & 132 & $50 \%$ & 115 \\
$\mathbf{5}$ & Nagpur & 8 & 16 & $50 \%$ & 55 \\
$\mathbf{6}$ & Jaipur & 98 & 202 & $48.5 \%$ & 100 \\
$\mathbf{7}$ & Kozikode & 7 & 15 & $46.7 \%$ & 30 \\
$\mathbf{8}$ & Kolkata & 55 & 121 & $45.5 \%$ & 61 \\
$\mathbf{9}$ & Bangalore & 11 & 29 & $37.9 \%$ & 63 \\
$\mathbf{1 0}$ & Mumbai & 25 & 122 & $20.5 \%$ & 63 \\
$\mathbf{1 1}$ & Guwahati & 1 & 8 & $12.5 \%$ & 49 \\
& Grand total & 386 & 842 & $45 \%$ & \\
\hline
\end{tabular}

b)

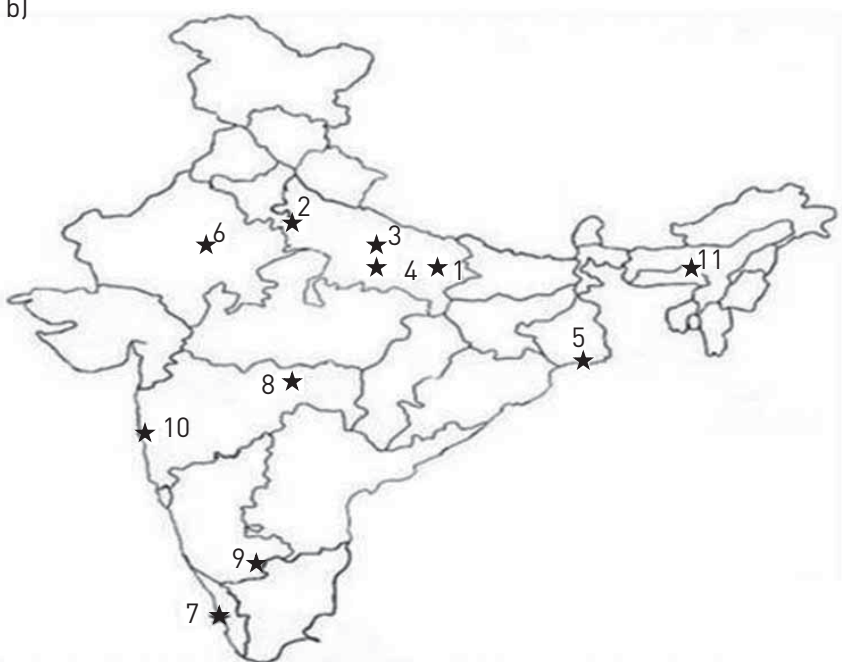

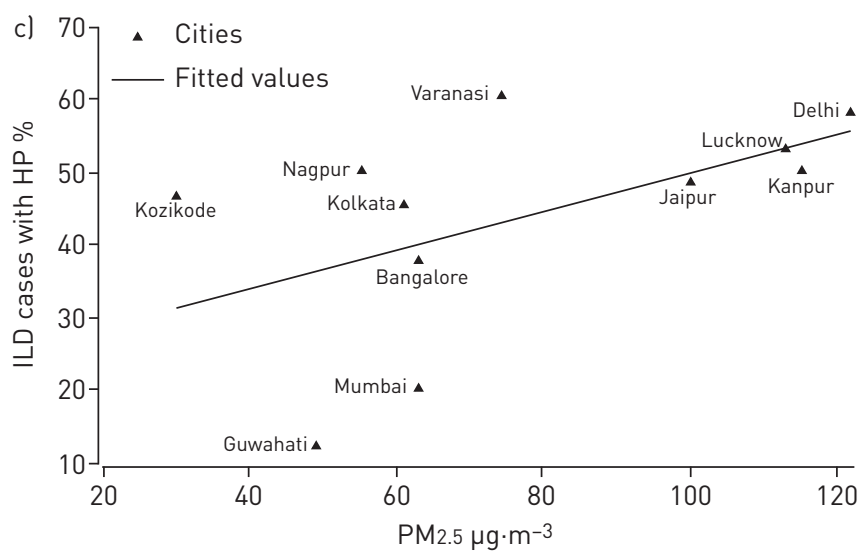

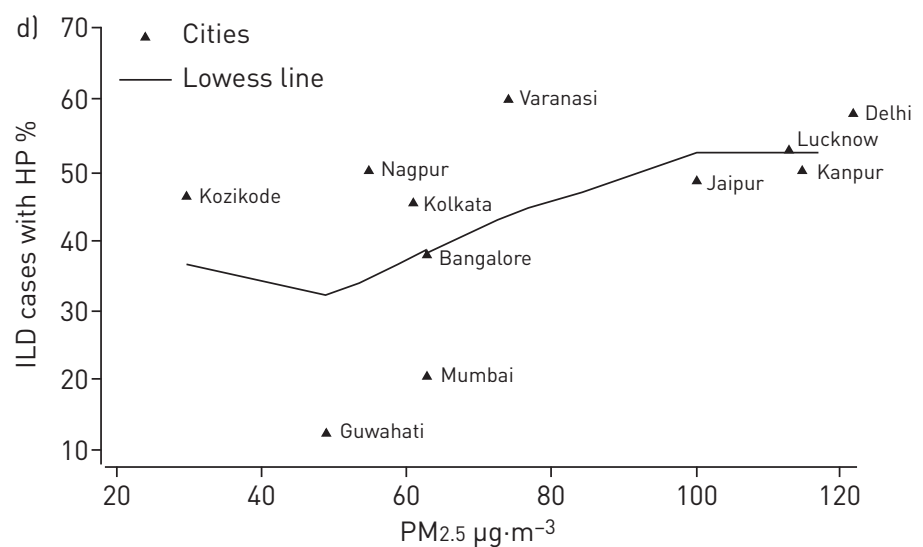

FIGURE 1 a) The 11 cities studied in the immune-mediated lung disease (ILD)-India registry with their percentage of hypersensitivity pneumonitis (HP) cases and particulate matter $<2.5 \mu \mathrm{m}$ in diameter (PM2.5) levels. b) Map of India showing the locations of the 11 cities. Scatter plots showing correlation between percent of HP cases in 11 cities and $\mathrm{PM}_{2.5}$ levels in that area with $\mathrm{c}$ ) best fitting line for linear regression and d) Lowess smoothing $(r=0.64, p=0.03)$

Spearman's rank correlation test revealed a strong positive correlation between the percentage of HP cases in the 11 cities and city PM2.5 levels $(\mathrm{r}=0.64, \mathrm{p}=0.03$ ) (figure $1 \mathrm{c}$ and $\mathrm{d}$ ). Multiple logistic regression analysis revealed that each $1 \mu \mathrm{g} \cdot \mathrm{m}^{-3}$ increase in PM2.5 level is associated with a $0.7 \%$ increase in the risk of developing HP rather than any other ILD (adjusted OR 1.007, 95\% CI 1.001-1.013; p=0.017). The sample sizes from patients with IPF and connective tissue-associated ILD from the same cities were too small to draw meaningful conclusions from similar analyses (data not shown).

Our findings raise the possibility of an association between HP diagnosis and environmental air pollution levels. It is apparent that air pollution may be a risk factor for patients to manifest HP similar to previous reports regarding viral infections and pesticide exposure $[3,4]$. Our results suggest that, although the confidence interval is wide, the odds of developing HP were $7 \%$ greater for every $10-\mu \mathrm{g} \cdot \mathrm{m}^{-3}$ increase in the air pollution (PM2.5 level). This association was adjusted for possible confounders such as birds, air conditioners, air coolers and moulds. It is apparent, therefore, that the risk of a person to manifest HP due to exposure to a known HP inducer, such as birds, is higher if the person has the same exposure and lives in urban areas. We hypothesise that air pollution is a possible contributing factor in the pathogenesis of HP; a potential mechanism is that fine particulate air pollutants lead to airway inflammation and reduced mucociliary clearance with resultant retention of the inciting antigen in the alveoli leading to an immune-mediated response and clinical manifestation of HP. Of note, ambient air pollution levels in India are significantly higher than those in other countries, including countries where many other ILD registries have been undertaken [8]. Since air pollution has been associated with worsening lung function status in patients with IPF [7], and has also been associated with poor outcomes in lung transplant recipients [9-11], the higher incidence of HP in patients enrolled in ILD-India registry greater than that observed in 
other registries may in part be attributed to differences in ambient air pollution levels. This will require further study both in India and other countries.

We acknowledge that our study is observational and the results are preliminary. Limitations of our findings include the following. 1) Our data are only from patients clinically diagnosed with HP residing in urban areas where PM2.5 levels were available (patients living in rural and remote areas were not included). 2) Nonuniform health coverage in India led to ILD registry sites being concentrated in urban cities. Thus, patients from rural areas were underrepresented, likely leading to referral bias. 3) It is uncertain whether the air monitors used to measure the PM2.5 levels were in close proximity to the patients' residences or workplaces. 4) Though multiple logistic regression was used to overcome biases due to other exposures, in a proportion of cases, the inciting agent is unknown and thus not accountable in statistical analysis. 5) PM2.5 levels were derived retrospectively from PM10 levels in the majority of cities from public sources, although derivation of PM2.5 levels from PM10 levels is considered to be fairly accurate [12].

Acknowledging these limitations, our observation that the proportion of new-onset ILD cases clinically diagnosed as HP increased with higher levels of air pollution in several Indian cities deserves further attention. We are hopeful that our results will provoke future studies with longitudinal designs, appropriate comparison groups and study participants that are selected from the population at risk to determine the clinical relevance of any potential association between HP and air pollution.

Sheetu Singh ${ }^{1}$, Bridget F. Collins ${ }^{2}$, Mohan Bairwa ${ }^{3}$, Jyotsna M. Joshi ${ }^{4}$, Deepak Talwar ${ }^{5}$, Nishtha Singh ${ }^{6}$, Jai K. Samaria ${ }^{7}$, Daya K. Mangal ${ }^{3}$, Virendra Singh ${ }^{6}$ and Ganesh Raghu ${ }^{2}$

${ }^{1}$ Dept Chest and Tuberculosis, SMS Medical College, Jaipur, India. ${ }^{2}$ Center for Interstitial Lung Diseases (CILD), Dept of Medicine, University of Washington, Seattle, WA, USA. ${ }^{3}$ Public Health and Epidemiology, IIHMR University, Jaipur, India. ${ }^{4}$ Dept of Pulmonary Medicine, Topiwala National Medical College and BYL Nair Hospital, Mumbai, India. ${ }^{5}$ Dept of Pulmonary and Sleep Care Medicine, Metro Multispeciality Hospital, Noida, India. ${ }^{6}$ Dept of Pulmonary Medicine, Asthma Bhawan, Jaipur, India. ${ }^{7}$ Dept of Chest Disease, Banaras Hindu University, Varanasi, India.

Correspondence: Ganesh Raghu, Center for Interstitial Lung Diseases, BOX 356175, University of Washington, Seattle, WA 98195, USA. E-mail id: graghu@uw.edu

Received: Aug 172018 | Accepted after revision: Oct 212018

Acknowledgements: We are grateful to the patients of ILD who consented to participate in the ILD India registry and indebted to all the site investigators Sandeep Katiyar (Chest Care Center, Kanpur, India), Parthasarthi Bhattacharya (Institute of Pulmocare and Research, Kolkata, India), Sudhir Chaudhari (GSVM Medical College, Kanpur, India), A.G. Ghoshal (National Allergy, Asthma, Bronchitis Institute, Kolkata, India), Gayathri Joshi (MS Ramaiah Medical College, Bangalore, India), Nirmal K. Jain (Jain Chest Care Center, Jaipur, India), Surya Kant (King George's Medical University, Lucknow, India), Raja Dhar (Fortis Hospital, Kolkata, India), Rajesh Swarnakar (Getwell Hospital and Research Institute, Nagpur, India), C. Ravindran (Calicut Medical College, Kozhikode, India), D.J. Roy (Pulmonary and Sleep Clinic, Kolkata, India), S.K. Sharma (AIIMS, New Delhi, India), K.R. Sarmah (Gauhati Medical College, Guwahati, India), Bhavin Jankharia (Jankharia Imaging, Mumbai, India), Uma Maheshwari (MS Ramaiah Medical College, Bangalore, India), Vivek Nangia (Fortis Hospital, New Delhi, India) and Rajesh Chawla (Indraprastha Apollo Hospital, New Delhi, India).

This study is registered at ctri.ni.in with identifier number CTRI/2013/05/003674.

Conflict of interest: None declared.

Support statement: The registry was partially funded by the Indian Chest Society, limited only to restricted, minimal expenses associated with mailing for data acquisition by the National Data Coordinating Center and sharing with Center for Interstitial Lung Diseases. Funding information for this article has been deposited with the Crossref Funder Registry.

\section{References}

1 Singh S, Collins BF, Sharma BB, et al. Interstitial lung disease in India. Results of a Prospective Registry. Am J Respir Crit Care Med 2017; 195: 801-813.

2 Selman M, Pardo A, King TE Jr. Hypersensitivity pneumonitis: insights in diagnosis and pathobiology. Am J Respir Crit Care Med 2012; 186: 314-324.

3 Dakhama A, Hegele RG, Laflamme G, et al. Common respiratory viruses in lower airways of patients with acute hypersensitivity pneumonitis. Am J Respir Crit Care Med 1999; 159: 1316-1322.

4 Hoppin JA, Umbach DM, Kullman GJ, et al. Pesticides and other agricultural factors associated with self-reported farmer's lung among farm residents in the Agricultural Health Study. Occup Environ Med 2007; 64: 334-342.

5 Cohen AJ, Brauer M, Burnett R, et al. Estimates and 25-year trends of the global burden of disease attributable to ambient air pollution: an analysis of data from the Global Burden of Diseases Study 2015. Lancet 2017; 389: $1907-1918$.

6 Lelieveld J, Evans JS, Fnais M, et al. The contribution of outdoor air pollution sources to premature mortality on a global scale. Nature 2015; 525: 367-371.

7 Winterbottom CJ, Shah RJ, Patterson KC, et al. Exposure to ambient particulate matter is associated with accelerated functional decline in idiopathic pulmonary fibrosis. Chest 2018; 153: 1221-1228. 
8 WHO Global Urban Ambient Air Pollution Database. 2016. www.who.int/phe/health_topics/outdoorair/databases/ cities/en/. Date last accessed: December 15, 2017.

9 Kaufman JD, Raghu G. Should we be concerned about air quality as a contributor to poor outcomes in lung transplant recipients? Eur Respir J 2017; 49: 1602369.

10 Benmerad M, Slama R, Botturi K, et al. Chronic effects of air pollution on lung function after lung transplantationin the Systems Prediction of Chronic Lung Allograft Dysfunction (SysCLAD) study. Eur Respir J 2017; 49: 1600206.

11 Ruttens D, Verleden SE, Bijnens EM, et al. An association of particulate air pollution and traffic exposure with mortality after lung transplantation in Europe. Eur Respir J 2017; 49: 1600484

12 Delfino RJ, Becklake MR, Hanley JA, et al. Estimation of unmeasured particulate air pollution data for an epidemiological study of daily respiratory morbidity. Environ Res 1994; 67: 20-38. 